Comparative Legilinguistics

vol. 30/2017

DOI : http://dx.doi.org/10.14746/cl.2017.30.3

\title{
LE PRÉAMBULE DE LA CONSTITUTION COMME UN EXEMPLE D'UN TEXTE DE DROIT
}

\author{
Piotr PIEPRZYCA \\ Pedagogical University in Kraków \\ Faculty of Language Studies \\ 30-084 Kraków, ul. Podchorążych 2 \\ ppieprzyca@gmail.com
}

Résumé : Le préambule de la Constitution est un type spécial de texte juridique qui diffère significativement des autres textes législatifs, en ce qui concerne le lexique, la syntaxe et la sémantique. Cet article vise à désigner les caractéristiques de la langue juridique du préambule de la Constitution - son contenu, sa forme et la fonction qu'il joue dans le système juridique. Le corpus de la recherche se compose des plus de vingt préambules des constitutions des pays européens. Les résultats montrent qu'en dépit de quelques différences entre les préambules des pays européens, il existe de nombreuses caractéristiques communes - presque tous les préambules mentionnent des valeurs et des principes, considérés comme fondamentaux pour le pays et la nation, tels que l'indépendance, la liberté ou la démocratie. Certains préambules se réfèrent aussi à l'histoire de son pays, à la religion ou à la personne de Dieu directement ou indirectement. Malgré le fait que le préambule - du point de vue linguistique - n'est pas comme les autres textes juridiques, il peut être considéré comme un texte normatif. 


\title{
Piotr Pieprzyca: Le Préambule de la Constitution ...
}

Mots-clés : préambule, constitution, langage juridique, norme juridique, linguistique juridique

\section{PREAMBULA KONSTYTUCJI JAKO PRZYKLAD TESKTU PRAWNEGO}

\begin{abstract}
Abstrakt: Preambuła konstytucji jest szczególnym rodzajem tekstu prawnego, różniącym się znacznie od pozostałych tekstów normatywnych, zarówno pod względem leksykalno-składniowym, jak i semantycznym. Niniejsza praca ma na celu dokonanie charakterystyki prawno-językowej preambuły konstytucji jej treści, formy oraz funkcji, jaką odgrywa w systemie prawnym. Korpus badawczy stanowią teksty ponad dwudziestu preambuł konstytucji krajów europejskich. Wyniki badań wskazują, że pomimo pewnych różnic pomiędzy preambułami poszczególnych krajów europejskich, istnieje wiele cech wspólnych - niemal wszystkie wymieniają bowiem wartości i zasady, postrzegane jako fundamentalne dla narodu, takie jak niezależność, wolność czy demokracja. Niektóre preambuły odwołują się również do historii swojego kraju lub do religii i samej osoby Boga - zarówno w sposób pośredni, jak I bezpośredni. Pomimo faktu, iż preambuła pod względem językowym nie przypomina innych tekstów prawnych, można przypisać jej cechy tekstu normatywnego.
\end{abstract}

Słowa kluczowe: preambuła, konstytucja, język prawny, norma prawna, legilingwistyka

\section{THE PREAMBLE TO THE CONSTITUTION AS AN EXAMPLE OF A LEGAL TEXT}

Abstract: Preamble to the Constitution is a special type of legal text, which differs significantly from the other legislative texts, both in terms of vocabulary, syntax and semantics. This paper aims to make the characteristics of the legal language of the preamble to the Constitution - its content, form and function it plays in the legal system. The linguistic corpus is composed of over twenty preambles to constitutions of European countries. The results show that, despite some differences between the preambles of individual European countries, there are many features in common - almost all mention the values and principles, considered as fundamental to the nation, such as independence, freedom or democracy. Some preambles also refer to the history of the country or religion and to the person of God - both indirectly and directly. Despite the fact that the preamble in terms of language is not like other legal texts, it has the characteristics of a normative act.

Key words: preamble, constitution, legal language, legal standard, legilinguistics 


\section{Introduction}

La constitution, appelée aussi loi fondamentale est un texte juridique qui se situe au sommet de la hiérarchie des actes normatifs. Elle a une valeur supérieure par rapport aux autres actes : lois, règlements, traités internationaux, etc. Parfois, pour souligner ce rang exceptionnel de la constitution, le législateur décide de précéder les règles qui s'y trouvent par une introduction solennelle connue sous le nom de préambule. Dans le présent article, nous allons caractériser le préambule, prenant en compte ses aspects linguistiques et juridiques. D'abord, nous présenterons la notion de préambule dans le contexte juridique (Point 2). Puis, nous décrirons le contenu (Chapitre 3) ainsi que la forme (Chapitre 4) du préambule afin de vérifier s'il a seulement une fonction décorative dans la constitution ou s'il a une valeur normative, comme la partie principale de la constitution qui contient des articles (Chapitre 5).

\section{La notion de préambule}

Selon le dictionnaire de français Larousse, le mot préambule a plusieurs sens:

1. Texte servant d'avant-propos, d'introduction et précédant un plus long développement ;

2. Propos, attitude ayant un lien plus ou moins direct avec le sujet et constituant une entrée en matière ;

3. Ce qui précède ou prépare ;

4. Partie préliminaire d'une constitution consistant en une proclamation solennelle des principes fondamentaux de l'organisation sociale ainsi que des droits et libertés des citoyens ;

5. Partie d'un traité qui énumère les hautes parties contractantes et les buts poursuivis (Définitions: préambule - Dictionnaire de français Larousse).

Les deux dernières définitions concernent la matière du droit. Elles nous indiquent que les préambules sont inclus seulement dans les constitutions et traités, mais, en fait ils sont aussi employés dans les 


\section{Piotr Pieprzyca: Le Préambule de la Constitution ...}

autres actes normatifs, par exemple, dans la loi polonaise sur la formation du système agricole ( $\mathrm{pl}$. Ustawa o kształtowaniu ustroju rolnego, Dz. U. z 2016 r. po.z 585, 1159) ou dans les actes juridiques communautaires (sous forme de visas et considérants).

Actuellement, une partie des constitutions des pays européens contient un préambule or pour d'autres le législateur a décidé d'omettre ce type d'introduction. C'est par exemple le cas de la constitution autrichienne ou hollandaise. Au total, seulement environ $50 \%$ des constitutions européennes ont un préambule. Parfois il est marqué explicitement par le titre Préambule ou par un autre titre (comme Profession de foi nationale dans la constitution hongroise ou Fondements historiques dans la constitution croate) ou sous la forme d'introduction sans aucun titre (comme dans la constitution grecque). Dans certains pays (p. ex. le Royaume-Uni ou la République de Saint Marin), le droit constitutionnel n'est pas formulé sous la forme d'un seul acte normatif, mais dans plusieurs lois qui le remplacent.

En s'appuyant sur le corpus composé d'environ 20 préambules des constitutions des pays européennes (mentionnés dans le tableau 1 et tableau 2), nous analyserons le préambule comme un type particulier de texte de droit sous l'angle de ses spécificités linguistiques et juridiques en essayant en même temps, de vérifier l'hypothèse selon laquelle on ne peut pas attribuer au préambule des caractéristiques spécifiques pour un texte de droit. Il faut souligner que notre analyse est fondée sur des textes français, donc dans le cas de pays francophones, ce sont les textes originaux mais en ce qui concerne les autres pays - ce sont les traductions disponibles sur le site Internet (Constitutions du Monde) ou sur les sites des gouvernements nationaux. Chaque pays a formulé son préambule d'une manière unique, prenant en compte plusieurs facteurs historiques, sociaux et même parfois religieux. Néanmoins, on va essayer de trouver quelques points communs dans les préambules des constitutions européennes, afin de les caractériser comme un type de texte qui n'est pas soumis au schéma d'un texte de droit.

\section{Le contenu du préambule}

Les préambules sont d'une longueur bien différente. Les plus courts sont les préambules des constitutions : française, monégasque et suisse (70-100 mots), le plus long est le préambule de la constitution 
hongroise (environ 700 mots). La longueur des préambules implique leur contenu - en général, plus la constitution est étendue, plus elle mentionne des valeurs.

La première phrase du préambule commence souvent par le nom peuple ou nation, éventuellement le pronom relatif nous remplace ces noms, comme par exemple :

\section{Exemple 1.}

(France) Le peuple français proclame solennellement son attachement aux droits de l'homme et aux principes de la souveraineté nationale (...)

\section{Exemple 2.}

(Tchéquie) Nous, citoyens de la République tchèque en Bohême, Moravie et Silésie (...)

\section{Exemple 3.}

(Espagne) La Nation espagnole, souhaitant établir la justice, la liberté et la sécurité et promouvoir le bien de tous ceux qui la composent, proclame, souverainement, sa volonté (...)

\section{Exemple 4.}

(Suisse) Le peuple et les cantons suisses (...)

Parfois le sujet se trouve à la fin du préambule :

\section{Exemple 5.}

(Estonie) Avec une foi infaillible et une volonté inflexible de maintenir et de développer l'État,

qui a été établi par le droit inaliénable du peuple estonien à l'autodétermination nationale et qui a été proclamé le 24 février 1918,

\section{(...)}

le peuple estonien a adopté par le référendum du 28 juin 1992, sur la base de l'article premier de la Constitution entrée en vigueur en 1938, la Constitution suivante.

C'est donc au nom du souverain - de la nation - que les législateurs formulent les préambules. On peut remarquer ici des traces de la philosophie du contrat social de Jean Jacques Rousseau, selon laquelle c'est la nation qui est la source du pouvoir d'Etat et c'est la nation qui établit la loi. 
Les deux principaux sujets des préambules sont l'histoire et les valeurs fondamentales.

La majorité des préambules contient des informations concernant les événements historiques marquant pour un pays donné. À cet égard, la plus étendue est la constitution croate. Dans son introduction, intitulée Fondements Historiques les faits historiques occupent presque la totalité du contenu. Le préambule de la constitution de la Serbie se focalise presque exclusivement sur le conflit dans l'histoire récente, c'est à dire le conflit de la région de KosovoMétochie.

Un autre sujet qui se répète dans presque tous les préambules et constitue leur contenu stricto sensu concerne les valeurs et les principes qui sont considérés comme fondamentaux et sont, pour cette raison, exposés dans le préambule. Ces principes forment un cadre axiologique du système juridique d'un pays.

Les tableaux 1 et 2 présentent les neuf valeurs les plus souvent mentionnées dans les textes des préambules européens comme: la liberté, l'indépendance, les droits de l'homme, le droit et la justice, la démocratie, la paix et la concorde, le progrès, la religion et l'environnement. 
Comparative Legilinguistics 2017/30

\begin{tabular}{|c|c|c|c|c|}
\hline PAYS & $\begin{array}{l}\text { Indépendance } \\
\text { / souveraineté }\end{array}$ & Liberté & $\begin{array}{c}\text { Droits } \\
\text { de l'homme }\end{array}$ & $\begin{array}{c}\begin{array}{c}\text { Droit et } \\
\text { justice }\end{array} \\
\end{array}$ \\
\hline Allemagne & + & + & & \\
\hline Andorre & + & + & + & + \\
\hline Biélorussie & + & + & + & + \\
\hline Croatie & + & + & + & + \\
\hline Espagne & + & + & + & + \\
\hline Estonie & + & + & & \\
\hline France & + & + & + & \\
\hline \multicolumn{5}{|l|}{ Grèce } \\
\hline Hongroie & + & + & + & + \\
\hline Irlande & + & + & + & + \\
\hline Lituanie & + & + & + & + \\
\hline Macédoine & + & + & + & + \\
\hline Moldavie & + & + & + & + \\
\hline Monaco & + & & & \\
\hline Pologne & + & + & + & + \\
\hline Portugal & + & + & + & + \\
\hline Russie & + & + & + & + \\
\hline \multicolumn{5}{|l|}{ Serbie } \\
\hline Suisse & + & + & & \\
\hline Tchèque & + & + & + & + \\
\hline Vatican & + & + & & \\
\hline Ukraine & + & + & + & + \\
\hline TOTAL & 20 & 19 & 15 & 14 \\
\hline
\end{tabular}

Tableau 1. Les valeurs mentionnées dans les préambules des constitutions européennes (I). 
Piotr Pieprzyca: Le Préambule de la Constitution ...

\begin{tabular}{|c|c|c|c|c|c|}
\hline PAYS & Démocratie & \begin{tabular}{|c|} 
Paix / \\
concorde
\end{tabular} & Progrès & Religion & Environnement \\
\hline Allemagne & & + & & + & \\
\hline Andorre & + & + & + & & + \\
\hline Biélorussie & & + & & & \\
\hline Croatie & + & + & + & & \\
\hline Espagne & + & + & & & \\
\hline Estonie & & + & + & & \\
\hline France & + & & & & + \\
\hline Grèce & & & + & & \\
\hline Hongroie & + & & & + & + \\
\hline Irlande & & + & & + & \\
\hline \multicolumn{6}{|l|}{ Lituanie } \\
\hline Macédoine & + & + & + & & \\
\hline Moldavie & + & + & + & & \\
\hline \multicolumn{6}{|l|}{ Monaco } \\
\hline Pologne & + & & & + & \\
\hline Portugal & + & & + & & \\
\hline Russie & + & + & & & \\
\hline \multicolumn{6}{|l|}{ Serbie } \\
\hline Suisse & + & & & + & \\
\hline Tchèque & + & & & & \\
\hline \multicolumn{6}{|l|}{ Vatican } \\
\hline Ukraine & + & + & + & + & \\
\hline TOTAL & 13 & 11 & 8 & 6 & 3 \\
\hline
\end{tabular}

Tableau 2. Les valeurs mentionnées dans les préambules des constitutions européennes (II). 
L'analyse du tableau permet de formuler quelques conclusions concernant le contenu des préambules.

En premier lieu l'indépendance et la liberté mentionnées par presque tous les préambules constituent deux principes fondamentaux. Ces valeurs sont fortement soulignées dans la constitution de la principauté d'Andorre, l'un des plus petits pays en Europe. La première phrase du préambule andorran exprime la fierté nationale des andorrans, résultant de l'indépendance :

Exemple 6.

(Andorre) Le peuple andorran, en pleine liberté et indépendance, et dans l'exercice de sa propre souveraineté,

La souveraineté est aussi fréquemment exposée au premier plan dans les préambules de pays qui, après la Seconde Guerre mondiale, ont été incorporés dans le bloc soviétique :

\section{Exemple 7.}

(Pologne) Soucieux de l'existence et de l'avenir de notre Patrie, ayant en 1989 recouvré la faculté de décider en toute souveraineté et pleine démocratie de notre destinée,

\section{Exemple 8.}

(Tchéquie) Nous, citoyens de la République tchèque en Bohême, Moravie et Silésie,

Au moment de la rénovation de l'État tchèque indépendant,

Quant à la deuxième valeur - la liberté, elle est aussi mentionnée dans presque tous les préambules aussi bien des pays les plus petits comme le Vatican que les plus grands comme la Russie.

Dans la constitution française, la liberté fait partie de la devise nationale, incluse dans le préambule :

\section{Exemple 9.}

(France) En vertu de ces principes et de celui de la libre détermination des peuples, la République offre aux territoires d'outre-mer qui manifestent la volonté d'y adhérer des institutions nouvelles fondées sur l'idéal commun de liberté, d'égalité et de fraternité et conçues en vue de leur évolution.

Presque $3 / 4$ des préambules se réfèrent aux droits de l'homme, p. ex. 
dans le préambule espagnol :

Exemple 10.

(Espagne) La Nation espagnole (...) proclame, souverainement, sa volonté de (...) protéger tous les Espagnols et tous les peuples d'Espagne dans l'exercice des droits de l'homme

La majorité des préambules parle aussi de la démocratie comme d'un fondement sur lequel s'appuie leur système juridique. Cela résulte $\mathrm{du}$ fait que presque tous les pays, à l'exception du Vatican (monarchie élective absolue) et du Liechtenstein, se définissent comme des pays démocratiques :

\section{Exemple 11.}

(Portugal) L'Assemblée constituante proclame la décision du peuple d'établir les principes de base de la démocratie,

\section{Exemple 12.}

(Ukraine) La Verkhovna Rada d'Ukraine, au nom du peuple ukrainien, les citoyens de l'Ukraine de toutes nationalités,

S'efforçant de développer et de renforcer une société démocratique, sociale, et un État fondé sur le droit ;

Les autres valeurs comme l'État de droit ou la paix sont aussi incluses dans la plupart des préambules européens. Dans le préambule de la loi fondamentale de la République fédérale d'Allemagne la paix est mentionnée au début du texte :

\section{Exemple 13.}

(Allemagne) Conscient de sa responsabilité devant Dieu et devant les hommes, animé de la volonté de servir la paix du monde

La constitution allemande a été créée en 1949, donc quatre ans après la Seconde Guerre mondiale. Le législateur avait probablement l'intention de déclarer de façon explicite que le nouveau système politique de l'Allemagne n'imitera pas le système précédent.

Dans seulement six constitutions on trouve une référence à Dieu (Allemagne, Pologne, Ukraine, Suisse, Irlande, Hongroie). Elle peut avoir deux formes différentes : 
1/ invocatio Dei - énoncé adressé directement à Dieu :

Exemple 14.

(Hongrie) Que Dieu bénisse les Hongrois !

\section{Exemple 15.}

(Suisse) Au nom de Dieu Tout-Puissant!

2/ nominatio Dei - énoncé qui parle de Dieu, mais elle n'est pas adressée directement à Dieu (Bała: 309):

\section{Exemple 16.}

(Pologne) nous, Nation polonaise - tous les citoyens de la République, tant ceux qui croient en Dieu,

source de la vérité, de la justice, de la bonté et de la beauté,

que ceux qui ne partagent pas cette foi et qui puisent ces valeurs universelles dans d'autres sources

La constitution grecque est la seule dont le préambule se compose seulement d'une phrase qui se réfère à la sainte Trinité :

\section{Exemple 17.}

(Grèce) Au nom de la sainte Trinité, consubstantielle et indivisible

Ce qui est assez étonnant c'est que la loi fondamentale du Vatican ne contient pas Invocatio Dei ou même Nominatio Dei dans le préambule ou même dans sa partie principale.

4. La forme du préambule

La langue employée dans les préambules est bien différente que celle dans laquelle sont formulés les actes normatifs, en ce qui concerne le lexique et la syntaxe.

Le caractère spécifique du préambule permet d'y employer des mots qui ne fonctionnent pas dans le langage du droit. Puisque les règles juridiques sont formulées à la troisième personne, les phrases à la première personne du pluriel sont absentes, contrairement au préambule. 
Les verbes déontiques qui servent à exprimer l'obligation ou l'interdiction ne sont pas non plus employés dans les préambules.

En ce qui concerne la structure syntaxique, l'ensemble du préambule est fréquemment formulé dans une seule phrase, dans laquelle les propositions sont séparées l'une de l'autre par une virgule, comme dans le préambule de la constitution de la Fédération russe :

\section{Exemple 18.}

(Russie) Nous, peuple multinational de la Fédération russe, unis par un destin commun sur notre terre,

proclamant les droits et les libertés de l'homme, la paix civile et la concorde, préservant l'unité de l'État forgée au fil de l'histoire,

conscients des principes universellement admis de l'égalité en droit et de l'autodétermination des peuples (...)

adoptons la Constitution de la Fédération russe.

De plus, le champ lexical des mots qui expriment les émotions est très développé, ce qui n'est pas souhaitable dans les articles des actes normatifs, par exemple : les sages traditions (Tchéquie), une foi infaillible, une volonté inflexible (Estonie), tournant historique (Portugal) l'amour et le respect de la Patrie (Russie) la loyauté, la foi et l'amour (Hongrie). Le législateur se réfère donc dans les préambules aux sentiments des destinataires.

En lisant la plupart des préambules inclus dans le corpus, on peut avoir l'impression que du point de vue linguistique, ils sont plus proches d'un texte littéraire que d'un texte juridique. En même temps il ne faut pas oublier qu'ils font partie des constitutions, des textes juridiques situés au sommet de la hiérarchie des actes normatifs.

\section{Le préambule a-t-il un caractère normatif ?}

Après avoir analysé le contenu et la forme du préambule, il faut enfin répondre à la question posée au début de la recherche : le préambule est-il un texte normatif ? La doctrine polonaise a développé trois conceptions différentes sur ce sujet. Selon la première - la plus radicale mais la moins courante - le préambule n'a pas de caractère normatif ou même juridique, mais avant tout politique (Skrzydło 2009 : 9). Selon la deuxième, le préambule n'a qu'une valeur interprétative. 
D'après la troisième conception, le préambule constitue un texte normatif, comme la partie principale de la constitution (Stefaniuk 2009 : 73-74).

Puisqu'il exprime les principes fondamentaux - sous la forme des dispositions et des obligations, de même que les articles, il semble que le préambule possède non seulement une valeur politique et interprétative, mais aussi normative. Cette hypothèse a été confirmée explicitement par le Tribunal Constitutionnel polonais dans sa décision K 2/07.

Dans la décision de K 34/97 de 3 juin 1998, le Tribunal a formulé une thèse selon laquelle la liberté, la justice, la coopération et le dialogue, comme valeurs fondamentales, mentionnées dans le préambule, doivent être respectées par les pouvoirs publics.

Le rôle du préambule devient particulièrement significatif au niveau juridique si les principes mentionnés dans le préambule sont absents dans la partie principale de la constitution (Stefaniuk 2005 : 205). Dans la constitution polonaise, c'est le principe de subsidiarité (décision no 29/00 du Tribunal Constitutionnel de 26 juin 2000).

Nous avons déjà observé que les préambules des constitutions européennes ont, sauf quelques exceptions, le même contenu, c'est à dire des valeurs, des principes et des buts qui doivent être poursuivis. On vient de constater que le préambule polonais peut être considéré comme un texte normatif, alors par analogie, il est légitime de généraliser pour dire que le préambule comme un type de texte est un texte qui a une valeur normative.

Bien sûr cette analyse n'est pas exhaustive en ce qui concerne la problématique du préambule. Pour mieux caractériser ce type de texte de droit, on a besoin d'effectuer des recherches avec un corpus plus étendu et une analyse plus complexe et approfondie. Néanmoins, cet article constitue un bon point de départ pour de prochaines recherches linguistiques en la matière.

La présente étude démontre aussi la problématique de la variété du langage juridique - il n'est pas uniforme et il a beaucoup de nuances qui sont toujours en attente de nouvelles études jurilinguistiques. 


\section{Bibliographie}

Bała, Paweł. 2016. Invocatio Dei w Konstytucji RP z 2 kwietnia 1997 r. w perspektywie porównawczej i historycznej, http://www.bibliotekacyfrowa.pl/Content/39049/016.pdf, consulté le 1 septembre 2016.

Définitions: préambule - Dictionnaire de français Larousse, http://www.larousse.fr/dictionnaires/francais/préambule/63282? q=preambule, consulté le 1 septembre 2016 .

Skrzydło, Wiesław. 2009. Konstytucja Rzeczpospolitej Polskiej. Komentarz. Warszawa : Wolters Kluwer Polska.

Stefaniuk, Małgorzata. 2005. Preambuła do Konstytucji Rp z 2 kwietnia 1997 roku w orzecznictwie Trybunału Konstytucyjnego. Annales Universitatis Mariae Curie-Skłodowska Lublin Polonia. Sectio G Ius. Vol. L/LI 2003/2004. ss. 203-223.

Stefaniuk, Małgorzata Ewa. 2009. Preambuła aktu normatywnego w doktrynie oraz $\mathrm{w}$ procesie stanowienia i stosowania polskiego prawa w latach 1989-2007. Lublin : Wydawnictwo Uniwersytetu Marii Curie-Skłodowskiej 2009.

\section{Décisions :}

Décision du Tribunal Constitutionnel de 11 mai 2007, $\mathrm{n}^{\mathrm{o}}$ K 34/97, otk.trybunal.gov.pl/orzeczenia/teksty/otk/2007/K_02_07.doc, consulté le 1 septembre 2016.

Décision du Tribunal Constitutionnel de 26 juin 2000, sign. 29/00. http://otk.trybunal.gov.pl/orzeczenia/teksty/otkpdf/2000/Ts029z 00.pdf, consulté le 1 septembre 2016.

Décision du Tribunal Constitutionnel de 3 juin 1997, $\mathrm{n}^{\circ}$ K 34/97, http://otk.trybunal.gov.pl/orzeczenia/teksty/otkpdf/1998/k_34_9 7.pdf, consulté le 1 septembre 2016. 


\section{Constitutions :}

Constitution de 1'Espagne, http://mjp.univ-perp.fr/constit/es1978.htm, consulté le 1 septembre 2016.

Constitution de l'Irlande, http://mjp.univ-perp.fr/constit/ie.htm, consulté le 1 septembre 2016.

Constitution de l'Ukraine, http://mjp.univ-perp.fr/constit/ua2011.htm, consulté le 1 septembre 2016.

Constitution de la Fédération de Russie, http://mjp.univperp.fr/constit/ru2008.htm, consulté le 1 septembre 2016.

Constitution de la Grèce, http://mjp.univ-perp.fr/constit/gr1975.htm, consulté le 1 septembre 2016.

Constitution de la Hongrie (officiellement nommée « Loi fondamentale de la Hongrie »), http://mjp.univ-perp.fr/constit/hu2011.htm, consulté le 1 septembre 2016.

Constitution de la Moldavie, http://mjp.univperp.fr/constit/md1994.htm, consulté le 1 septembre 2016.

Constitution de la Principauté l'Andorre, http://mjp.univperp.fr/constit/and1993.htm, consulté le 1 septembre 2016.

Constitution de la République d'Estonie, http://mjp.univperp.fr/constit/ee1992.htm, consulté le 1 septembre 2016.

Constitution de la République de Biélorussie, http://mjp.univperp.fr/constit/by1996.htm, consulté le 1 septembre 2016.

Constitution de la République de Croatie, http://mjp.univperp.fr/constit/hr.htm, consulté 1 septembre 2016.

Constitution de la République de Lituanie, http://mjp.univperp.fr/constit/lt1992.htm, consulté le 1 septembre 2016.

Constitution de la République de Macédoine, http://mjp.univperp.fr/constit/mk1991.htm, consulté le 1 septembre 2016.

Constitution de la République de Pologne, http://www.sejm.gov.pl/prawo/konst/francuski/kon1.htm, consulté le 1 septembre 2016.

Constitution de la République du Portugal, http://mjp.univperp.fr/constit/pt1976.htm, consulté le 1 septembre 2016.

Constitution de la République fédérale d'Allemagne, https://www.bundestag.de/blob/189762/f0568757877611b2e434 039d29a1a822/loi_fondamentale-data.pdf, consulté le 1 septembre 2016. 
Piotr Pieprzyca: Le Préambule de la Constitution ...

Constitution de la République française, http://mjp.univperp.fr/france/co1958.htm, consulté le 1 septembre 2016.

Constitution de la République Tchèque, http://mjp.univperp.fr/constit/cz1992.htm, consulté le 1 septembre 2016.

Constitution de la Serbie, http://mjp.univ-perp.fr/constit/rs2006.htm, consulté le 1 septembre 2016.

Constitution de Monaco, http://mjp.univ-perp.fr/constit/mon1962.htm, consulté le 1 septembre 2016.

Constitution fédérale de la Confédération suisse, http://mjp.univperp.fr/constit/ch2000.htm, consulté le 1 septembre 2016.

Constitutions du Monde, http://mjp.univ-perp.fr/constit/constitintro.htm, consulté le 1 septembre 2016.

Loi fondamentale de l'État de la Cité du Vatican, http://mjp.univperp.fr/constit/va2001.htm, consulté le 1 septembre 2016. 